\title{
Effects of Cranial Radiation on Structural and Functional Brain Development in Pediatric Brain Tumors
}

\author{
Jesse C. Bledsoe ${ }^{1}$
}

Received: 10 June 2015 / Revised: 28 September 2015 / Accepted: 1 October 2015 /Published online: 19 October 2015

(C) American Academy of Pediatric Neuropsychology 2015

\begin{abstract}
There is emerging research connecting neuroimaging correlates and neurocognitive functioning in pediatric brain tumors. Current work has attempted to measure the impact of cranial radiation on brain and behavior outcomes with the goal of better understanding the mechanisms of treatment late effects. While critical for treatment, cranial radiation can have a substantial impact on the developing brain and on neuropsychological development. This paper provides a critical review of the most recent empirical structural and functional neuroimaging research evaluating the impact of cranial radiation on neuropsychological functioning in pediatric brain tumors. The importance in study methodology, potential outcome moderating variables, limitations, and future directions is discussed.
\end{abstract}

Keywords Brain tumor · Medulloblastoma $\cdot$ Late effects . Neurocognitive $\cdot$ Neuroimaging

\section{Introduction}

Brain tumors are the leading cause of cancer-related death in pediatric oncology, second only to leukemia in childhood malignancy, and account for approximately $18 \%$ of newly diagnosed pediatric cancers (Howlader et al. 2013). Brain tumors in the posterior fossa (i.e., medulloblastomas and ependymomas) are the most common and account for around $30 \%$ of pediatric brain tumors (Ostrom et al. 2014). Standard treatment

Jesse C. Bledsoe

bledsoe1@washington.edu

1 Department of Psychiatry and Behavioral Medicine, University of Washington School of Medicine, Seattle Children's Hospital, 4800 Sand Point Way NE, OA.5.154, Seattle, WA 98105, USA procedures often include maximal surgical resection of the tumor, radiation therapy, and adjuvant chemotherapy (Gottardo and Gajjar 2006). Due to the aggressive nature of these treatments as well as damage to the brain from the tumor, many survivors experience debilitating neurological, neuropsychological, and psychosocial outcomes. Cranial radiation treatment, which is highly effective at destroying cancerous cells and preventing relapse for many types of tumors, is also associated with neurocognitive late effects: a developmental plateau or decline in age-expected cognitive development, observed as early as 1 year posttreatment. Younger age at diagnosis and higher radiation dosage has been associated with worse neurocognitive outcomes (Kieffer-Renaux et al. 2000; Merchant et al. 2006). Some studies have observed as high as 15 point reductions in intellectual functioning (i.e., intellectual quotient or IQ) and deficits in reading, memory, cognitive functioning, attention, and processing speed (Mulhern et al. 2005; Palmer et al. 2001). Moreover, some children will experience such an impact on their functioning that they will no longer be able to live independently as adults. While the exact mechanism of neurocognitive late effects is unknown, there is mounting evidence to suggest that radiation may impede normal development of both white matter and gray matter. However, these findings are mixed, and the effects of radiation on brain-behavior relationships remain equivocal.

There are many variables, independent from radiation that have a direct impact on neurocognitive development and should be considered when discussing and evaluating late effects of treatment. For example, direct impact from the tumor, hydrocephalus and shunt revisions, posterior fossa syndrome, prolonged absence from school, and psychosocial changes are all side effects of brain tumor treatments and exert their unique and multiplicative impact on the cognitive, academic, and psychological development of individual survivors. The purpose of this review is to provide background 
on the causal mechanisms of late effects, discuss the relationship between neuroimaging outcomes and neurocognitive outcomes, and evaluate potential outcome moderating variables in neurocognitive functioning in pediatric brain tumors.

\section{Literature Reviewed and Inclusion Criteria}

Literature review and search were completed with both Ovid MEDLINE and PsycINFO. Search terms included the following: pediatric brain tumors, medulloblastoma, ependymoma, cranial radiation, irradiation, neurocognitive, neurocognitive late effects, functional magnetic resonance imaging (fMRI), functional neuroimaging, structural neuroimaging, cortical thickness, cortical volume, white matter, gray matter, diffusion tensor imaging (DTI), fractional anisotropy, and computed tomography (CT) (Fig. 1). There were several studies evaluating the impacts of radiation on neurocognitive functioning though the purpose of this review was to evaluate literature that included both neuroimaging and neurocognitive methods. Studies that employed only neurocognitive or neuropsychological measures were excluded. Case studies or studies with sample sizes of five or fewer were also excluded. There were a total of 18 peer-reviewed studies that met these inclusion criteria for this review.

\section{Radiation and the Developing Brain}

In general, children treated with radiation at younger ages are most vulnerable to developing cognitive late effects. The

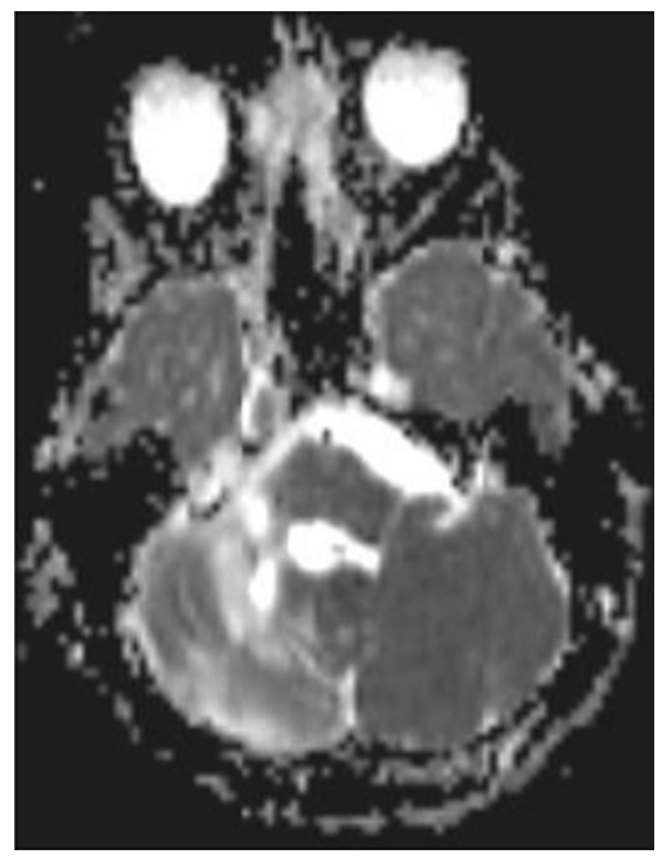

Fig. 1 Axial DTI ADC of cerebellar ependymoma. DTI diffusion tensor imaging, $A D C$ apparent diffusion coefficient. Figure produced by the author reason for this is closely connected to the developmental trajectory of individual cortical gray matter and subcortical white matter. In order to understand the potential mechanism behind cognitive late effects, it is important to discuss the typical pattern of gray and white matter development.

Human brain development is characterized by an overproduction and then elimination (i.e., pruning) of cells and a remarkable balance between cell proliferation and cell death (Huttenlocher 1990). It is this protracted period of production and pruning that is at the core of neurocognitive development and learning. Brain volume increases significantly during the first 5 to 6 years of life. This tremendous rate of growth is due to the proliferation of neuroglial cells as well as increased myelination (Huttenlocher 1979). Anatomically, the brain can be divided into gray matter (cortex) and white matter (subcortical axons). Gray matter consists of neuronal cell bodies which are responsible for the conduction of neural activity. While different depending on anatomical structure, gray matter follows an inverted $U$-shaped curve peaking in volume around age 4 and then slowly declining across the life span (Giedd et al. 1999). White matter, the brain's myelinated axons, responsible for connecting neuronal synapses, increases almost linearly throughout childhood and adolescence with peak myelination occurring in the middle 20s (Giedd et al. 1996; Huttenlocher 1979). Rapid white matter myelination occurs in the first few years of life and also presents a particularly vulnerable period for injury (Huttenlocher 1999). It is during these early years of synaptogenesis, pruning, and myelination that ionizing radiation treatments are thought to have the most deleterious effects on the developing brain.

\section{Neuroanatomical Mechanisms of Radiation Late Effects}

The primary mechanism of radiation late effects is damage to gray matter and white matter via oxidative stress and inflammation, angiogenesis, and neuronal cell death. Clinically, late effects may be observed on magnetic resonance imaging (MRI) as multifocal damage in white matter and gray matter. However, regional white matter volume and networks of white matter integrity are the most commonly studied structures that have been evaluated in pediatric brain tumors.

\section{White Matter Structure and Function}

The majority of neuroimaging studies on the neuroanatomical effects of radiation have involved white matter volume, area, and estimates of the integrity of white matter connectivity (see Table 1). Some of the earliest work on white matter changes following treatment for medulloblastoma sought to compare radiological readings of atrophy and estimates of gray matter 
Table 1 Common neuroimaging techniques

Technique Image acquisition

Use in pediatric brain tumors

MRI Introduction of a magnetic field first aligns hydrogen nuclei (protons) on a plane. Radio waves are then introduced and pulsed in order to alter the spatial orientation of the nuclei out of the aligned plane. As the nuclei drift back into their original aligned plane, they emit a detectable signal that produces a structural image.

fMRI The premise of fMRI is the detection of cerebral blood flow in active and functional regions of the brain. fMRI uses a blood-oxygen level dependent signal (BOLD) that is produced by the ratio of deoxygenated-oxygenated blood.

Because MRI does not use ionizing radiation it is considered a safe neuroimaging procedure for children. Also, MRI produces both $\mathrm{T} 1$ and $\mathrm{T} 2$ images which allow for improved imaging of tumor location and tumor type.

Technique for determining the functionality of cerebral vasculature and the potential location of activation during a task. fMRI does not require the injection of radioactive isotopes (like positron emission tomography or single photon emission computed tomography) making it a safe procedure for children.

DWI Same principle as DTI, however, DWI allows for the assessment of apparent diffusion coefficient (ADC) maps.

DWI and ADC can be used to detect cellularity which provides information regarding tumor grade. May also provide validation of histological analysis. ADC ratios may also differentiate between tumor progression vs. radiation injury.

DTI Detects the diffusion of water (nonrandom anisotropic movement) in Est white matter fiber tracts compared to random (Brownian) movement in other brain tissues.

Estimates the strength/integrity of white matter axonal pathways in the brain. Fractional anisotropy (FA) may be useful for predicting tumor grade, differentiate between infiltrative tumor tissue and vasogenic edema, and healthy tissue. The visualization of white matter fiber tracks relative to tumor tissue may help with neurosurgical planning.

$M R I$ magnetic resonance imaging, $f M R I$ functional magnetic resonance imaging, $D T I$ diffusion tensor imaging, $D W I$ diffusion weighted imaging, $A D C$ apparent diffusion coefficient, $F A$ fractional anisotropy

and white matter volumes assessed by quantitative MRI (Reddick et al. 1998). Initial findings suggested brain parenchyma and white matter volumes declined with increased atrophy, though no relationship between gray matter volume and atrophy was observed. After further dividing their dataset based on treatment approach, they observed no measurable effect of chemotherapy on white matter volume, gray matter volume, or brain parenchyma. The groups were then reduced to those treated with radiation and surgery versus those treated with surgery alone. As hypothesized, at 1-year posttreatment, all children treated with radiation showed significantly reduced white matter volumes. It was observed later that estimates of what the authors called normal white matter (NWM; based on volumetric MRI estimation of segmented brain tissue) were lower in children with medolloblastoma treated with surgical resection and cranial radiation (23-36 Gy with boost to 4954 Gy) compared to an age-matched sample of children with low-grade astrocytoma (LGA) treated with surgical resection alone (Mulhern et al. 1999). They also found the medulloblastoma group performed significantly lower than the LGA group on performance IQ and full-scale IQ (FSIQ) estimates and that all IQ estimates (full scale, performance, and verbal) were significantly and positively correlated with normal white matter volume in both the medulloblastoma and LGA groups. The findings suggest a brain-behavior relationship supporting the hypothesis that white matter and overall neurocognitive function was negatively affected by irradiation.

In a similar study, patients with medulloblastoma were found to have roughly 1 to 1.5 standard deviations below average full-scale IQ, nonverbal IQ, verbal memory, and sustained attention abilities (Mulhern et al. 2001). Deficits in all neurocognitive domains except verbal memory were positively correlated with estimates of NWM volume. After controlling for age at radiation treatment and time since radiation treatment, the authors suggest that roughly $70 \%$ of the relationship between age at radiation treatment and IQ was attributed to an estimated volume of NWM. They concluded that the relationship between white matter development and neurocognitive functioning was mediated by age at radiation treatment, a finding that was subsequently replicated in a larger sample (Reddick et al. 2005). These authors have also observed significant correlations between NWM matter and fullscale IQ $(r=.32)$ and attention abilities $(r=.49)$ (Reddick et al. 2003). However, after controlling for attention and memory ability (verbal learning), the correlation between NWM and IQ diminished, and the authors suggest that attention ability and memory were moderating variables. It is important to point out that the relationship between attention measures and NWM was not appreciated in previous reports (Mulhern et al. 1999). This could have been due to differences in sample characteristics (e.g., age at treatment or diagnoses) or possibly reflect a finding that needs further replication.

Another potential question is whether white matter development is moderated by radiation dose. While most studies used clinical samples receiving similar radiation protocols (20-40 Gy with boost to 50-60 Gy), only a few to date have examined the effects of total radiation dose on white matter volumes with mixed results. One study observed $23 \%$ slower rate of NWM volume loss in children treated with reduced radiation dose (23.4 Gy vs. conventional 36 Gy whole brain) 
(Reddick et al. 2000). However, it is unclear if those receiving reduced radiation dose had inherent differences in brain tumor characteristics or treatments, which may have accounted for white matter differences. This represents a central challenge inherent to evaluating radiation dosage effects. In contrast, Palmer et al. (2002) observed abnormalities in posterior corpus callosum development in children treated for medulloblastoma, though no differences were observed when patients were stratified by dose (23.4 vs. 36-39.6 Gy). While it is possible that these early deviations in posterior corpus callosum area were due to radiation treatment, especially given the regions prolific growth in early childhood (Giedd et al. 1996), the study failed to detect a dose effect, and therefore, the issue deserves further study.

The results from these early studies clearly implicated white matter abnormality as a potentially etiological explanation for neurocognitive late effects, though the impact of radiation dose on cognitive outcome is not clear. Future work will need to carefully evaluate the impact of radiation dose while attempting to control for tumor type, use consistent methodology, and use age-matched controls in order to provide valid and replicable results.

\section{Diffusion Tensor Imaging and White Matter Integrity}

More recent work has utilized diffusion-weighted (DWI) and diffusion tensor imaging (DTI) techniques to evaluate the late effects of radiation on white matter structure and integrity (Figs. 2 and 3). Common research outcomes/scalers of DWI and DTI include fractional anisotropy (FA), apparent diffusion coefficient (ADC), and mean diffusivity (MD). FA value is a quantity based on the diffusion of water molecules along a specified white matter axon bundle (e.g., 0 isotropic and 1 for complete anisotropic diffusion) and is considered an estimate of white matter density or extent of myelination. ADCs are depictions of the strength of water diffusion based on the ratio of low signal intensity due to high water diffusion and high signal intensity due to low water diffusion. MD is an estimation of the average diffusion within a given voxel or specified fiber bundle.

DTI and DWI are the most commonly used neuroimaging metrics for evaluating white matter impairments in pediatric brain tumors (see Table 1). In general, studies have observed decreased FA values in several white matter networks including frontal and parietal periventricular white matter, corpus callosum, corona radiata, right inferior fronto-occipital fasciculus, anterior and posterior limbs of the internal capsule, inferior frontal white matter, high frontal white matter, pons, medulla oblongata, and cerebello-thalamo-cerebral tracts (Aukema et al. 2009; Khong et al. 2003; Khong et al. 2005; Law et al. 2011; Leung et al. 2004; Mabbott et al. 2005).

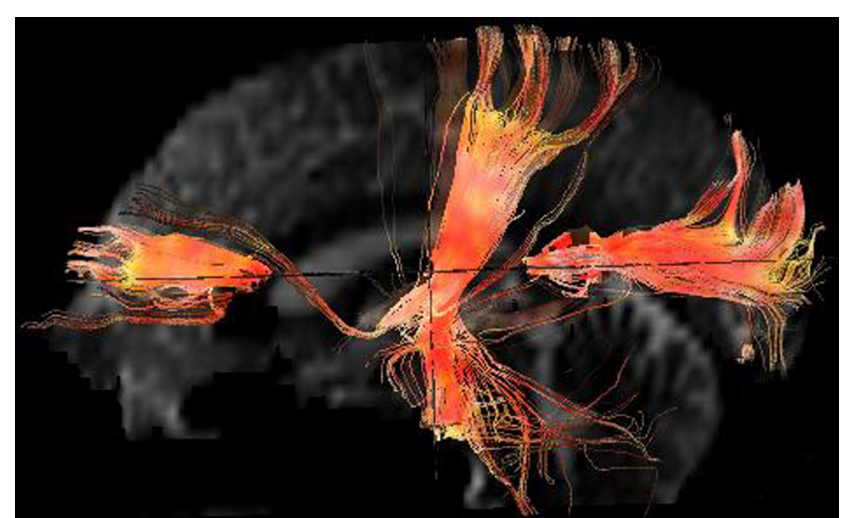

Fig. 2 Diffusion tensor image fiber track (3T)—-sagittal. DTI diffusion tensor imaging, $3 T 3$ tesla. Figure produced by the author

Diffuse and nonfocal increases in ADC have also been implicated in those treated with high doses of cranial radiation (Law et al. 2011; Mabbott et al. 2005). It is clear from these studies that white matter integrity is compromised in a wide range of frontal and posterior white matter projections. Furthermore, there is some evidence that posterior white matter may be more sensitive to disruption in younger children due to the relative posterior-anterior development of the brain.

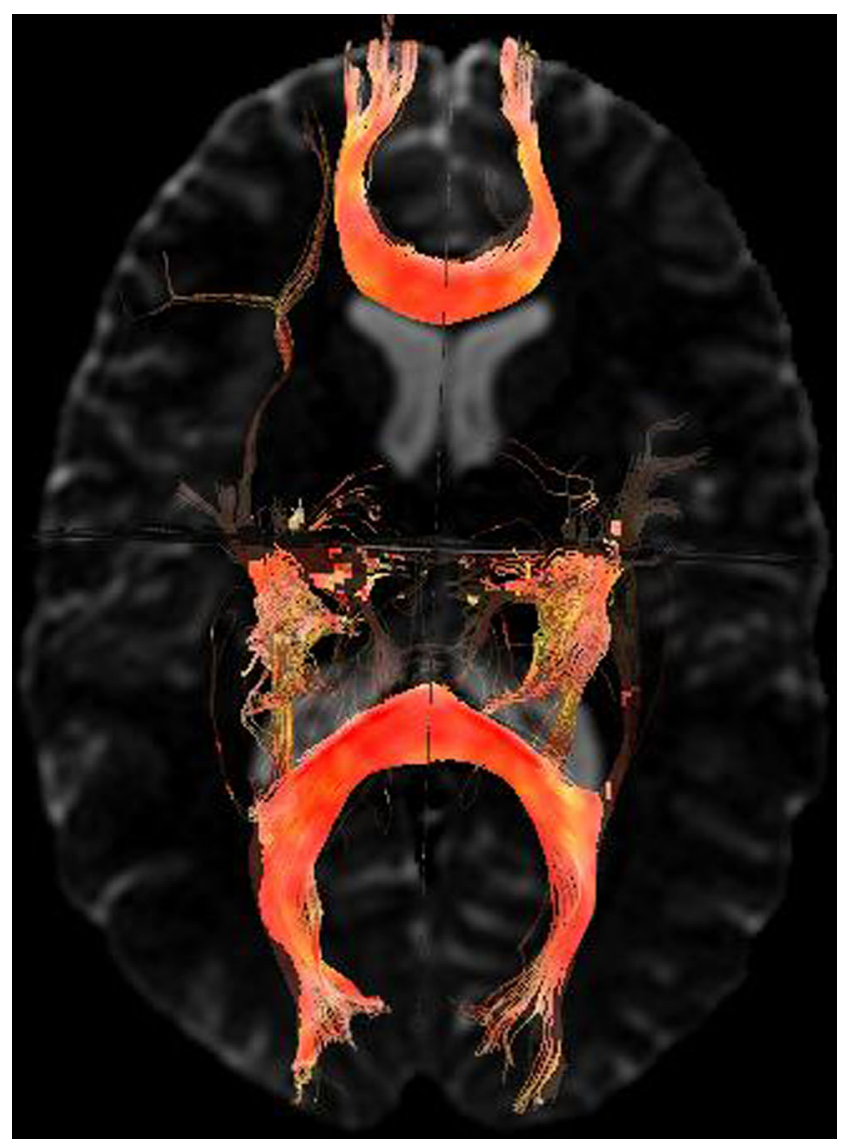

Fig. 3 DTI fiber track (3T) - axial. DTI diffusion tensor imaging, 3T 3 tesla. Figure produced by the author 
There are several methodological limitations of these studies that preclude interpretations about individual brain structures. For example, many studies reported using age-matched controls in an effort to account for developmental differences in white matter volume. This is critical factor given the effect of age on white matter volume and diffusion characteristics (Barnea-Goraly et al. 2005). However, most studies compared patient and control groups of different ages, do not report control group age ranges, or did not include a control group from which to compare white matter volume changes. Studies also included samples with vast age ranges (e.g., 3-19 years old) further complicating the interpretation of statistical "means" which might not adequately be statistically controlled for given the inherent relationship (i.e., correlation) between age and dynamic brain structural development. Given the relatively small number of children diagnosed with brain tumors, it remains challenging to match subjects on age at treatment, age at testing, and exact treatment protocols and still obtain sample sizes large enough to detect statistically meaningful results. Nonetheless, future studies will require appropriate use of age-matched controls and careful control of age effects (i.e., radiation, neurocognitive testing) in order to improve the accuracy, validity, and clinical utility of results.

\section{Diffusion Tensor Imaging and Function}

White matter damage is also thought to play a causal role in the development of neurocognitive side effects in children with brain tumors. Several studies have observed correlations between white matter diffusion properties and numerous neurocognitive domains (see Table 1). The most studied, and therefore observed, neurocognitive effects in the literature is that of overall or composite measures of intellectual functioning. The reason is that measures of overall intellectual functioning are considered part of the standard clinical neuropsychological battery for neuropsychologists evaluating treatment effects on cognitive functioning, not because the measures have been found to be sensitive or specific to treatment late effects. The results regarding overall IQ estimates and their relationship to white matter changes are mixed. For example, Aukema et al. (2009) observed differences in processing speed measures from the Dutch WISC-IV though no differences in full-scale IQ. Others have observed nonsignificant differences in mean FSIQ in a sample of children with medulloblastoma compared to the standardization mean (Palmer et al. 2010). In contrast, others have observed nearly 1 standard deviation below the normative mean in children medulloblastoma (Brinkman et al. 2012), though the authors report no correlation between IQ and estimates of FA, ADC, or radial diffusivity. In a similar study, Mabbott et al. (2005) reported a significant correlation between mean FSIQ measures and FA values. However, this finding was not significant until an outlier was removed from the dataset. It should also be stated that the control group FSIQ was in the high average range (112, corresponding to the 79th percentile) and the mean for the medulloblastoma group was in the average range (95.25) and did not statistically differ from that of the control group (prior to removal of one outlier). Thus, the interpretation that white matter abnormality is functionally related to changes in, or "low," overall intellectual functioning needs further study and should be carefully evaluated in the existing literature.

More task-specific neurocognitive domains have also been studied. For example, information processing speed, as measured by the Dutch Wechsler Intelligence Scale for Children 3rd Edition (Dutch WISC-III), and fine-motor control were positively correlated with FA values in the splenium of the corpus callosum and inferior frontal-occipital fasciculus, respectively, in children with medulloblastoma compared to controls (Aukema et al. 2009). This was a somewhat surprising finding given that the full-scale intelligence measure (FSIQ) and perceptual reasoning composite scores for the control group were both in the low average range. Others have observed differences in FA values on reading performance (i.e., pseudoword decoding) in patients with brain tumors when stratified by "above average" or "below average" readers (Palmer et al. 2010). It was unclear how the authors defined above- vs. below-average readers, as the means and standard deviations were not reported. The authors also mention using two measures of reading performance from the Woodcock-Johnson 3rd Edition though only the raw scores from the Word Attack subtest are reported. Future studies will need to carefully evaluate the impact of white matter abnormalities and their relationship to academic skill development. Various executive functioning tasks have also been implicated in white matter abnormalities including RD and shifting attention, cognitive flexibility, and working memory (Brinkman et al. 2012; Law et al. 2011). Taken together, there does not appear to be a clear pattern of white matter abnormalities and associated neurocognitive relationships in pediatric brain tumors. Instead, findings suggest diffuse white matter differences and nonspecific neurocognitive differences in those treated for brain tumors (Table 2).

\section{Gray Matter}

The most common methods for analyzing gray matter morphology involve measuring regional volume or cortical thickness. Several early studies comparing white matter and gray matter morphology found decreases in "normal white matter" and no differences in gray matter volume (Reddick et al. 2005; Reddick et al. 1998; Reddick et al. 2000; Reddick et al. 2003). It is unclear if these investigations included different participants or if some participants were included in several studies 





(i.e., all from St. Jude Children's Research Hospital) which would present a significant confound. Using estimates of cortical thickness as opposed to volumetric measures, Liu et al. (2007) observed thinning in the left parietal-occipital cortex, and right parietal, posterior superior temporal, and lateral temporal cortex compared to healthy controls. The authors postulate that these parietal and temporal regions were likely affected, while frontal regions were spared due to the more posterior regions' vulnerability at the time of radiation. This finding was not replicated in a more recent study and may have been due to differences in the age ranges at imaging or possibly differences in cortical thickness analysis procedures (Brinkman et al. 2012). For example, Brinkman et al. (2012) do not report on the extent of editing of white and gray matter boundaries, whether they conducted regional or whole brain analysis, how they analyzed cortical thickness estimates, and on the software used to process the data. These potential differences in methodology could explain the differences between these studies. Radiation treatment has also been associated with initial hippocampal volume reductions 2-3 years after treatment (Nagel et al. 2004). However, hippocampal volume measurements were also related to gender, level of parental education, shunt placement, and seizure history. This provides evidence that radiation may not be a sufficient explanation for gray matter changes in children with brain tumors.

\section{Functional Magnetic Resonance Imaging}

Task-based MRI is used to evaluate regional cortical activation (blood oxygen level-dependent signal; BOLD) during a specific and carefully defined behavioral tasks (Fig. 4). To date, only two controlled studies have been published in the field of pediatric brain tumors using fMRI. In a recent study, BOLD signal activation during a cognitive working memory task ( $n$-back test) was different in survivors of pediatric brain tumors compared to controls (Robinson et al. 2014). Brain tumor survivors recruited greater activation in the left dorsal anterior cingulate cortex compared to controls. Group differences in cognitive and executive functioning were corroborated by neuropsychological measures of intellectual functioning (Wechsler Intelligence Scale for Children-4th Edition; WISC-IV composites) (Wechsler 2003), on two executive functioning subtests (Delis-Kaplan Executive Function System; D-KEFS inhibition/switching and trails letter/number switching) (Delis et al. 2001), and on parent report questionnaires (Child Behavior Checklist; CBCL attention problems and Behavioral Ratings Inventory of Executive Function; BRIEF) (Achenbach 1991). While measures were statistically different from healthy controls, only letter/number switching from the D-KEFS and the processing speed index from the WISC-IV were in the "low average" range. Thus, we must be cautious not to label brain tumor survivors' as having "deficits" 


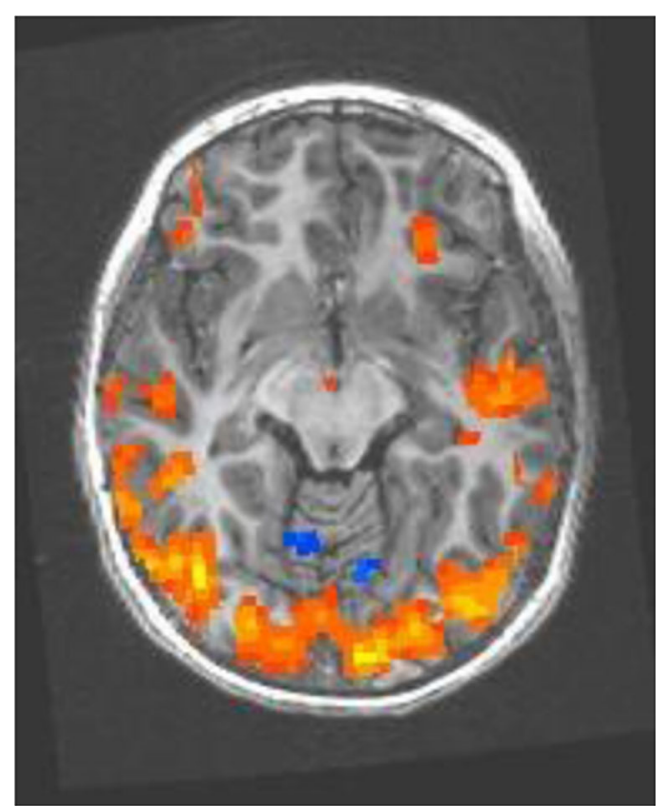

Fig. 4 Blood oxygen level dependent signal (BOLD) activation during task-based fMRI. Figure produced by the author

when the vast majority of scores were well within the average range compared to the standardization mean. This is particularly pertinent when the control group mean is in the high average range. In another fMRI study utilizing an $n$-back working memory paradigm, the authors observed BOLD activation clusters in the right inferior frontal gyrus, supplementary motor area, bilateral insula, bilateral inferior parietal lobule, and right middle occipital gyrus (Wolfe et al. 2013). Though there was no control group, the authors report that BOLD activation in these regional clusters is consistent with previous work and therefore indicates "normal" working memory activation patterns. Interestingly, the authors employed a measure of cardiorespiratory fitness which was associated with improved working memory performance. The results suggest that cardiorespiratory fitness may moderate executive functioning in survivors of pediatric brain tumors. This finding has been corroborated in studies across the lifespan (Antsey et al. 2004).

\section{Potential Moderating Variables}

One of the central limitations inherent in the most studies on neurocognitive late effects is the multitude of variables that can moderate neurocognitive outcomes. In the current literature, there is an assumption that a brain tumor, the treatments, and putative brain structure differences have a direct impact on individual neurocognitive development. While this hypothesis is likely, it is unclear what percent of the actual variance in neurocognitive outcomes is accounted for by the tumor, the treatments, or other factors known to have a direct impact on cognitive and academic development. Future work that attempts to account for as many factors moderating individual cognitive ability will improve our understanding and measurement of late effects. The factors reviewed here are not exhaustive, though representing a good starting point for ongoing work.

\section{Hydrocephalus and Shunt Revision}

Hydrocephalus affects many children with brain tumors, particularly those undergoing second surgery, and has a known deleterious effect on neurocognitive function. Side effects from hydrocephalus can be longstanding and include motor difficulties, visual/auditory difficulties, decreased intellectual functioning, special education, and behavior disorders (Hoppe-Hirsch et al. 1998). The extent of hydrocephalus has been found to moderate the impact of radiation therapy on IQ as well as verbal and nonverbal abilities (Brookshire et al. 1995; Merchant et al. 2004). Furthermore, increased intracranial pressure preoperatively and symptom duration have been found to be an important predictor of long-term intellectual function (Yule et al. 2001). Given the impacts of hydrocephalus on neurocognitive outcomes, further evaluation of the role of hydrocephalus as a moderating variable in studies of neurocognitive late effects is critical. In some studies, the percent of patients with hydrocephalus is reported, though this is not the norm. Coding either the absence or presence of hydrocephalus has been found to be inadequate at determining how it affects patient's neurocognitive outcomes. Instead, a more quantitative assessment (e.g., ventricular volume, Evan's index, cella media index, frontal horn diameter, and ventricular angle) is recommended as it is considered a more sensitive and predictive method for evaluating the impact of hydrocephalus on neurocognitive outcomes (Merchant et al. 2004).

\section{Posterior Fossa Syndrome}

A large percentage of brain tumors (e.g., medulloblastoma and ependymoma) develop in the posterior fossa region located between the cerebellum and brainstem nuclei. Damage to white matter connections within the cerebellar-thalamiccerebral network during surgical resection can cause posterior fossa syndrome, a clinical syndrome characterized by significant speech deficits including loss of expressive language (Morris et al. 2009). The duration of speech difficulties associated with surgery can last from weeks to over a year with varying and nonlinear levels of language recovery. Neurocognitive profiles in children with posterior fossa syndrome can vary widely and may include dysarthria, aphasia, executive functioning difficulty, and academic impairments 
(Catsman-Berrevoets and Aarsen 2010; Palmer et al. 2010). Taken together, posterior fossa syndrome is a clinical syndrome that affects a significant proportion of children treated for brain tumors and has demonstrated impacts on neurocognitive functioning over 12 months after treatment. Given the varying levels of deficits observed in posterior fossa syndrome, this represents an important confound in many of the studies reviewed here. Future studies correlating structural and functional imaging data with neurocognitive outcomes should include posterior fossa syndrome as a clinical variable that can be controlled for statistically, evaluated separately, or analyzed with regard to the $\%$ variance explained in individual datasets.

\section{Prolonged School Absence/Learning Environment}

One significant confound in the study of treatment late effects is the assumption of equal environments in patient groups compared to controls. Children treated for brain tumors may spend weeks or possibly years in treatment and in recovery. For many children, this equates to a significant time away from school, decreased opportunities for cognitive development and learning, financial burden that may impact a family's ability to provide typical enriched experiences, as well as physical, social, and emotional challenges. Given the robust relationship between educational attainment, socioeconomic resources, and the enriched environment on intellectual and cognitive functioning (Ceci 1991; Turkheimer et al. 2003), and on developing neurocircuitry (Noble et al. 2012), future studies should attempt to measure these potential moderators of neurocognitive outcomes.

\section{Radiation Dose}

One assumption in the literature is that radiation dose moderates structural and functional brain development and also neurocognitive development. Radiation dosage is generally reported in the literature as the total dose to the tumor site and is typically between 50 and $60 \mathrm{~Gy}$. Other estimates of radiation dose, including dose per fraction (typically 1.8 $2.0 \mathrm{~Gy}$ ) and number of radiation treatment days during the week, are less commonly reported in the literature. Radiation may have a dose-dependent impact on some neurocognitive domains (Merchant et al. 2006), though it is still unclear what specific domains are sensitive to radiation as individuals may differ considerably in their neurocognitive functioning both before and after radiation treatment. There is some evidence that those treated with lower doses of radiation may demonstrate areas of better neurocognitive functioning than those treated with higher doses (Mabbott et al. 2005; Merchant et al. 2004; Mulhern et al. 2005). However, less is known about how differing levels of radiation affect brain structure and function given that most of the studies evaluating brain and behavior relationships include only participants with total doses above $50 \mathrm{~Gy}$. In one study, white matter volume loss was $23 \%$ slower in children receiving 23.4 Gy compared to those receiving 36 Gy (Reddick et al. 2000); however, dose effects were not observed when evaluating corpus callosum area estimates in a longitudinal design (Palmer et al. 2002). Radiation dose effects on both brain and behavior outcomes are clearly in need of more careful study. Furthermore, dose effects could be moderated by age at treatment or age at neurocognitive assessment, and so, future studies will need to attempt to address radiation dose as a significant moderator of results in their methods and statistical analysis.

\section{Individual Differences}

The vast majority of research reviewed attempts to measure the statistical difference between two groups (e.g., brain tumor group vs. control group). While this is meaningful for understanding group level differences, this statistical approach provides very little information regarding individual outcomes, therefore reducing the clinical utility of the findings. For example, the group mean on neurocognitive variables for patients with medulloblastoma in several studies was in the average or low average range with a range of 1-2 standard deviations. This suggests that a potentially large proportion of patients with medulloblastoma performed within (or above) the standardization and control group average range. However, this is overshadowed by common methods such as univariate and less often multivariate statistics (e.g., $t$ tests, analysis of variance, and general linear model). Future work may address this issue by either describing their findings at the group level (percentage of patients performing in the average range) or by using new statistical techniques such as machine learning/support vector machine (SVM) algorithms to determine individual differences in neurocognitive outcomes with established sensitivity and specificity. SVM is being evaluated to help detect individual differences with disease progression, diagnosis, and treatment in psychiatric disease (Orru et al. 2012). Such techniques may provide useful information for individual classification of neurocognitive late effects, prognosis, and potentially moderate treatment.

\section{Conclusions}

We provide a review of 18 empirical studies evaluating brain and behavior relationships in children treated for brain tumors. There are both conclusions as well as questions that can be derived from this body of work. First, there is evidence that age at the time of radiation treatment may moderate the impact 
on white matter development, with children below age 6-7 experiencing greater impacts. It can also be concluded that white matter abnormalities, if observed, are diffuse and nonfocal. Second, it is unclear if the amount of treatment has a dose-dependent impact on white matter development in individuals. This hypothesis was tested in several studies and is inconclusive and in need of replication with increased sample sizes and carefully diagnosed and age-matched control participants. Third, the relationship between white matter abnormalities and neurocognitive functioning is mixed and suggests nonspecific neurocognitive difficulties relative to peers. In many studies, patients with brain tumors performed within the average range relative to the standardization mean on neurocognitive measures though may have performed lower than a control group. Fourth, there are many variables that can moderate brain-behavior relationships in this line of research. For example, history of shunt revision/hydrocephalus, absence from school due to illness, posterior fossa syndrome, and radiation dose may be potential moderators of outcome. These variables have a known impact on neurocognitive function and should be evaluated in future studies in order to provide a clear and accurate depiction of the impact of treatments on neurocognitive and academic outcomes. Furthermore, there is vast heterogeneity regarding neuroanatomical and neurocognitive outcomes within individuals treated for brain tumors. This heterogeneity is difficult to capture with current models of treatment outcome and statistical analysis of group data. We propose future work that aims to develop neurocognitive phenotypes that will better reflect individual differences. The study and development of neurocognitive phenotype models may also provide better understanding of underlying neuroanatomical differences in children based on individual differences. Such work would ultimately allow for targeted neuropsychological assessment to measure outcomes, specific cognitive remediation strategies, and possibly inform medical treatment, at the level of individuals.

\section{Compliance with Ethical Standards}

Conflict of Interest The author declares that he has no conflict of interest. No animal or human studies were carried out by the author for this article.

Informed Consent Informed consent was not applicable to this review article.

\section{References}

Achenbach, T. M. (1991). Manual for the Child Behavior Checklist and Revised Child Behavior Profile. Burlington, VT: T.M. Achenbach.

Antsey, K. J., Windsor, T. D., Jorm, A. F., Christensen, H., \& Rodgers, B. (2004). Association of Pulmonary Function with Cognitive
Performance in Early, Middle and Late Adulthood. Gerontology, 50(4), 230-234.

Aukema, E. J., Caan, M. W. A., Oudhuis, N., Majoie, C. B., Vos, F. M., Reneman, L., et al. (2009). White matter fractional anisotropy correlates with speed of processing and motor speed in young childhood cancer survivors. International Journal of Radiation Oncology and Biol Phys., 74(3), 837-843.

Barnea-Goraly, N., Menon, V., Eckert, M., Tamm, L., Bammer, R., Karchemskiy, A., et al. (2005). White matter development during childhood and adolescence: a cross-sectional diffusion tensor imaging study. Cerebral Cortex, 15(12), 1848-1854.

Brinkman, T. M., Reddick, W. E., Luxton, J., Glass, J. O., Sabin, N. D., Srivastava, D. K., et al. (2012). Cerebral white matter integrity and executive function in adult survivors of childhood medulloblastoma. Neuro-Oncology, 14, iv25-iv36.

Brookshire, B. L., Fletcher, J. M., Bohan, T. P., Landry, S. H., Davidson, K. C., Francis, D. J. (1995). Verbal and nonverbal skill descrepancies in children with hydrocephalus: a five-year longitudinal study. Journal of Pediatric Psychology, 20(6), 785-800.

Catsman-Berrevoets, C. E., \& Aarsen, F. K. (2010). The spectrum of neurobehavioral deficits in the Posterior Fossa Syndrome in children after cerebellar tumor surgery. Cortex, 46(7), 933-946.

Ceci, S. J. (1991). How much does schooling influence general intelligence and its cognitive components? A reassessment of the evidence. Developmental Psychology, 27(5), 703-722.

Delis, D. C., Kaplan, E., \& Kramer, J. H. (2001). Delis-Kaplan executive functioning system: examiner's manual. San Antonio, TX: The Psychological Corporation.

Giedd, J., Blumenthal, J., Jeffries, N. O., Castellanos, F. X., Liu, H., Zijdenbos, A., et al. (1999). Brain development during childhood and adolescence: a longitudinal MRI study. Nature Neuroscience, 2(10), 861-863.

Giedd, J., Rumsey, J., Castellanos, F. X., Rajapakse, J. C., Kaysen, D., Vaituzis, A. C., et al. (1996a). A quantitative MRI study of the corpus callosum in children and adolescents. Brain Research. Developmental Brain Research, 26(91), 274-280.

Giedd, J., Snell, J. W., Lange, N., Rajapakse, J. C., Casey, B. J., Kozuch, P. L., et al. (1996b). Quantitative magnetic resonance imaging of human brain development: ages 4-18. Cerebral Cortex, 6, 551-559.

Gottardo, N. G., \& Gajjar, A. (2006). Current therapy for medulloblastoma. Current Treatment Options Neurologyl, 8, 4.

Hoppe-Hirsch, E., Laroussinie, F., Brunet, L., Sainte-Rose, C., Renier, D., Cinalli, G., et al. (1998). Late outcome of the surgical treatment of hydrocephalus. Child's Nervous System, 14, 97-99.

Howlader, N., Noone, A. M., Krapcho, M., Garshell, J., Neyman, N., Altekruse, S. F., et al. (2013). SEER Cancer Statistics Review, 1975-2010. Bethesda, MD: National Cancer Institute.

Huttenlocher, P. R. (1979). Synaptic density in human frontal cortex Developmental changes and effects of aging. Brain Research, 163(2), 195-205.

Huttenlocher, P. R. (1990). Morphometric study of human cerebral cortex development. Neuropsychologia, 28(6), 517-527.

Huttenlocher, P. R. (1999). Dendritic and synaptic development in human cerebral cortex: time course and critical periods. Developmental Neuropsychology, 16(3), 347-349.

Khong, P. L., Kwong, D., Chan, G. C. F., Sham, J., Chan, F., \& Ooi, G. (2003). Diffusion-tensor imaging for the detection and quantification of treatment-induced white matter injury in children with medulloblastoma: a pilot study. American Journal of Neuroradiology, 24, 734-740.

Khong, P. L., Leung, L. H. T., Chan, G. C. F., Kwong, D., Wong, W., Cao, G., et al. (2005). White matter anisotropy in childhood medulloblastoma survivors: association with neurotoxicity risk factors. Radiology, 23, 647-652.

Kieffer-Renaux, V., Bulteau, C., Grill, J., Kalifa, C., Viguire, D., \& Jambaque, I. (2000). Patterns of neuropsychological deficits in 
children with medulloblastoma according to craniospinal irradiation doses. Developmental Medicine and Child Neurology, 42(11), 741745

Law, N., Bouffet, E., Laughlin, S., Laperriere, N., Briere, M., Strother, D., et al. (2011). Cerebello-thalamo-cerebral connections in pediatric brain tumor patients: impact on working memory. NeuroImage, $56,223-2248$.

Leung, L. H. T., Ooi, G., Kwong, D., Chan, G. C. F., Cao, G., \& Khong, P. L. (2004). White-matter diffusion anisotropy after chemo-irradiation: a statistical parametric mapping study and histogram analysis. NeuroImage, 21, 261-268.

Liu, A. K., Marcus, K. J., Fischl, B., Grant, P. E., Poussaint, T. Y., Rivkin, M., et al. (2007). Changes in cerebral cortex of children treated for medulloblastoma. International Journal of Radiation Oncology and Biol Phys., 68(4), 992-998.

Mabbott, D. J., Spiegler, B. J., Greenberg, M. L., Rutka, J. T., Hyder, D. J., \& Bouffet, E. (2005). Serial evaluation of academic and behavioral outcome after treatment with cranial radiation in childhood. Journal of Clinical Oncology, 23(10), 2256-2263.

Merchant, T. E., Kiehna, E. N., Li, C., Shukla, H., Sengupta, S., Xiong, X., et al. (2006). Modeling radiation dosimetry to predict cognitive outcomes in pediatric patients with CNS embryonal tumors including medulloblastoma. International Journal of Radiation Oncology and Biol Phys., 65(1), 210-221.

Merchant, T. E., Lee, H., Zhu, J., Xiong, X., Wheeler, G., Phipps, S., Sanford, R.A. (2004). The effect of hydrocephalus on intelligence quotient in children with localized infratentorial ependymoma before and after focal radiation therapy. Journal of Neurosurgery, 2(101), 159-168.

Merchant, T. E., Mulhern, R. K., Krasin, M. J., Kun, L., Williams, T., Li, C., et al. (2004). Preliminary results from a phase II trial of conformal radiation therapy and evaluation of radiation-related CNS effects for pediatric patients with localized ependymoma. Journal of Clinical Oncology, 1(22), 3156-3162.

Morris, E. B., Phillips, N. S., Laningham, F. H., Patay, Z., Gajjar, A., Wallace, D., et al. (2009). Proximal dentatothalamocortical tract involvement in posterior fossa syndrome. Brain, 132(11), 30873095.

Mulhern, R. K., Palmer, S. L., Merchant, T. E., Wallace, D., Kocak, M., Brouwers, P., et al. (2005). Neurocognitive consequences of riskadapted therapy for childhood medulloblastoma. Journal of Clinical Oncology, 23(24), 5511-5519.

Mulhern, R. K., Palmer, S. L., Reddick, W. E., Glass, J. O., Kun, L. E., Taylor, J., et al. (2001). Risk of Young Age for Selected Neurocognitive Deficits in Medulloblastoma Are Associated With White Matter Loss. Journal of Clinical Oncology, 19(2), 472-479.

Mulhern, R. K., Reddick, W. E., Palmer, S. L., Glass, J. O., Elkin, T. D., Kun, L. E., et al. (1999). Neurocognitive Deficits in Medulloblastoma Survivors and White Matter Loss. Annals of Neurology, 46(6), 834-841.

Nagel, B. J., Palmer, S. L., Reddick, W. E., Glass, J. O., Helton, K., Wu, S., et al. (2004). Abnormal hippocampal development in children with medulloblastoma treated with risk-adapted irradiation. American Journal of Neuroradiology, 25, 1575-1582.

Noble, K. G., Houston, S. M., Kan, E., \& Sowell, E. R. (2012). Neural correlates of socioeconomic status in the developing human brain. Developmental Science, 15(4), 516-527.
Orru, G., Pettersson-Yeo, W., Marquand, A. F., Sartori, G., \& Mechelli, A. (2012). Using support vector machine to identify imaging biomarkers of neurological and psychiatric disease: a critical review. Neuroscience and Biobehavioral Reviews, 36, 1140-1152.

Ostrom, Q. T., Gittleman, H., Liao, P., Rouse, C., Chen, Y., Dowling, J., Ostrom, Q. T., Gittleman, H., Liao, P., Rouse, C., Chen, Y., Dowling, J., et al. (2014). CBTRUS Statistical Report: primary brain and central nervous system tumors diagnosed in the United States in 2007-2011. Neuro Oncology, 16(s5), iv1-iv63.

Palmer, S. L., Goloubeva, O., Reddick, W. E., Glass, J. O., Gajjar, A., Kun, L., et al. (2001). Patterns of intellectual development among survivors of pediatric medulloblastoma: a longitudinal analysis. Journal of Clinical Oncology, 19(8), 2302-2308.

Palmer, S. L., Hassall, T., Evankovich, K., Mabbott, D. J., Bonner, M., Deluca, C., et al. (2010). Neurocognitive outcome 12 months following cerebellar mutism syndrome in pediatric patients with medulloblastoma. Neuro-Oncology, 12(12), 1311-1317.

Palmer, S. L., Reddick, W. E., Glass, J. O., Gajjar, A., Goloubeva, O., \& Mulhern, R. K. (2002). Decline in corpus callosum volume among pediatric patients with medulloblastoma: longitudinal MR imaging study. American Journal of Neuroradiology, 23, 1088-1094.

Reddick, W. E., Glass, J. O., Palmer, S. L., Wu, S., Gajjar, A., Langston, J. W., et al. (2005). Atypical white matter volume development in children following craniospinal irradiation. Neuro-Oncology, 7(1), $12-19$.

Reddick, W. E., Mulhern, R. K., Elkin, T. D., Glass, J. O., Merchant, T. E., \& Langston, J. W. (1998). A hybrid neural network analysis of subtle brain volume differences in children surviving brain tumors. Magnetic Resonance Imaging, 16(4), 413-421.

Reddick, W. E., Russell, J. M., Glass, J. O., Xiong, X., Mulhern, R. K., Langston, J. W., et al. (2000). Subtle white matter volume differences in children treated for medulloblastoma with conventional or reduced dose craniospinal irradiation. Magnetic Resonance Imaging, 18, 787-793.

Reddick, W. E., White, H. A., Glass, J. O., Wheeler, G. C., Thompson, S. J., Gajjar, A., et al. (2003). Developmental model relating white matter volume to neurocognitive deficits in pediatric brain tumor survivors. Cancer, 97(10), 2512-2519.

Robinson, K. E., Pearson, M. M., Cannistraci, C., Anderson, A. W., \& Kuttesch, J. (2014). Neuroimaging of executive function in survivors of pediatric brain tumors in healthy controls. Neuropsychology, 28(5), 791-800.

Turkheimer, E., Haley, A., Waldron, M., D'Onofrio, B., \& Gottesman, I. I. (2003). Socioeconomic status modifies heritability of IQ in young children. Psychological Science, 14(6), 623-628.

Wechsler, D. (2003). Wechsler Intelligence Scale for Children (WISC-IV) (4th ed.). San Antonio, TX: The Psychological Corporation.

Wolfe, K. R., Madan-Swain, A., Hunter, G. R., Reddy, A. T., Banos, J., \& Kana, R. K. (2013). An fMRI investigation of working memory and its relationship with cardiorespiratory fitness in pediatric posterior fossa tumor survivors who received cranial radiation therapy. Pediatric Blood and Cancer, 60(4), 669-675.

Yule, S. M., Hide, T. A. H., Cranney, M., Simpson, E., Barrett, A. (2001). Low grade astrocytomas in the West of Scotland 1987-96: treatment, outcome, and cognitive functioning. Archives of Disease in Childhood, 84, 61-64. 